\title{
Division X: Radio Astronomy
}

\author{
PRESIDENT: Luis F. Rodríguez \\ VICE-PRESIDENT: Ren-Dong Nan \\ ORGANIZING COMMITTEE: Philip J. Diamond, Gloria Dubner, \\ Michael Garrett, Anne Green, Masato Ishiguro, W. Miller Goss, \\ Russ Taylor, Lucia Padrielli (deceased), A. Pramesh Rao, \\ José M. Torrelles, and Jean L. Turner
}

\begin{abstract}
There have been important advances in radio astronomy in the last three years. New discoveries both at the galactic and extragalactic scale have been reported over this period and we highlight here several of them. The outstanding results of the Wilkinson Microwave Anisotropy Probe satellite, allowing an accurate determination of the main cosmological constants, are certainly among the most important. At the international level, the consolidation of the Atacama Large Millimeter Array project, with participation of the USA, Europe, and Japan and an estimated cost of around one billion US dollars, takes the construction of radio telescopes to a new level of complexity and potential. We also include the Progress Report of the Working Group on Historic Radio Astronomy, that includes a description of the duties and activities of this recently created working group.
\end{abstract}

\section{Introduction}

During the past triennium, radio astronomy witnessed many changes and improvements in the available facilities. Perhaps the major event at the international level was the consolidation and initiation of the Atacama Large Millimeter Array project. In the page http://www.astrosmo.unam.mx/IAU_Com40/2005/facilities.html we have included links to all major radio astronomical facilities where the details of the recent instrumental developments can be consulted.

The scientific results have continued to flow with great continuity. In the first section of this document we have listed some of these results that sample the great variety of research being undertaken. By far, this report is not complete and only tries to convey the vitality and versatility of the field.

We also note with sadness two deceases in the period considered. Grote Reber, that with Karl Jansky was considered one of the pioneers of radio astronomy, died in Tasmania on 2002 December 20, just two days short of his 91st birthday. Lucia Padrielli, the past President of Division X passed away on 2003 December 22. Lucia was President of our Division during the period 2000-2003 and a distinguished researcher and academic leader, both at the italian and international levels.

\section{Scientific Highlights June 2002-June 2005 (Luis F. Rodríguez)}

\subsection{Our Galaxy}

\subsubsection{The Sun and Stars}

For a summary of the type of radio observations of the Sun been carried out in recent years, we refer the reader to section 2.5.3 of Trimble \& Aschwanden (2004).

Dougherty et al. (2005) were able to follow the radio emission in the wind-collision region of the archetype $\mathrm{W}-\mathrm{R}+\mathrm{O}$ star colliding-wind binary system WR 140. They find 
that the region is a bow-shaped arc that rotates as the orbit progresses and are able to model it.

Using VLBI observations, Loinard et al. (2005) have made very precise (2\%) determinations of the trigonometric parallax of $\mathrm{T}$ Tau South. This technique could be extended to other gyro-synchrotron stars in regions of star formation.

\subsubsection{Molecular Clouds and Star Formation}

A recent review on ultra-compact H II regions and massive star formation has been made by Churchwell (2002). An update of more recent results is presented in Rodríguez (2005).

Observing at millimeter wavelengths, Harvey et al. (2002) and Shirley et al. (2002) have studied the density structure of molecular cores to test the "inside-out" collapse models. A detailed chemical study of the molecular core in 25 transitions of 9 molecules was presented by Evans et al. (2005).

Patel et al. (2005) presented images and kinematical data of a disk of dust and molecular gas around a high-mass protostar. This result is important because it has been proposed that high-mass stars form through accretion of material from a circumstellar disk, in essentially the same way as low-mass stars form. However, the alternative possibility that high-mass stars form through the merging of several low-mass stars should be further explored. Along these lines, the observations of proper motions in the BN object and the I source in the Orion KL region suggest the possible formation of a close binary or even a merger after a three-body encounter between young massive stars (Rodríguez et al. 2005).

Glycine, the simplest of amino acids, may have been detected by Kuan et al. (2003) using an improved search strategy for intrinsically weak molecular lines. However, Snyder et al. (2005) have questioned the detection arguing that several key lines necessary for a rigorous interstellar Glycine identification have not yet been found.

\subsubsection{Pulsars and Supernovae}

Lyne et al. (2004) reported the detection of the 2.8-second pulsar J0737-3039B as the companion to the 23-millisecond pulsar J0737-3039A in a highly relativistic double neutron star system. It is expected that this true binary pulsar system will allow unprecedented tests of fundamental gravitational physics.

Hankins et al. (2003) report the discovery of isolated, highly polarized, two-nanosecond subpulses within the giant radio pulses from the Crab pulsar. The plasma structures responsible for these emissions must be smaller than one meter in size, making them by far the smallest objects ever detected and resolved outside the Solar System, and the brightest transient radio sources in the sky.

Ransom et al. (2005) identified 21 new millisecond pulsars in the globular cluster Terzan 5, bringing the total of known such objects in Terzan 5 to 24 . These discoveries confirm fundamental predictions of globular cluster and binary system evolution.

Cameron et al. (2005) detected a radio counterpart to the 27 December 2004 giant flare from SGR 1806-20 and were able to obtain a high-resolution 21-cm radio spectrum that traces the intervening interstellar neutral hydrogen clouds. Analysis of this spectrum yields the first direct distance measurement of SGR 1806-20: the source is located at a distance greater than $6.4 \mathrm{kpc}$ and the authors argue that it is nearer than $9.8 \mathrm{kpc}$. If correct, this distance estimate lowers the total energy of the explosion and relaxes the demands on theoretical models.

Chatterjee et al. (2005) measured the proper motion and parallax for the pulsar $\mathrm{B} 1508+55$, leading to model-independent estimates of its distance $(2.37 \pm 0.22 \mathrm{kpc})$ and transverse velocity $\left(1083 \pm 100 \mathrm{~km} \mathrm{~s}^{-1}\right)$. This is the highest velocity directly measured for a neutron star. 


\subsubsection{Microquasars}

The microquasar GRS $1915+105$ has been studied exhaustively and the combination of radio and $\mathrm{X}$ ray data has advanced our understanding or the coupling between inflow (in an accretion disk) and outflow (in the relativistic jets) in this source (Fender \& Belloni 2004). Marscher et al. (2002) find in the galaxy 3C120 that, as has been observed in microquasars, the dips in the X-ray emission are followed by ejections of bright superluminal knots in the radio jet. This result points to an accretion-disk origin for the radio jets in active galaxies.

\subsubsection{The Galactic Center}

The studies of the linear and circular polarizations associated with Sgr A* are expected to reveal crucial information with regard to the radio source (Bower et al. 2002; 2004). Shen et al. (2005) report a radio image of Sgr $\mathrm{A}^{*}$ at a wavelength of $3.5 \mathrm{~mm}$, demonstrating that its size is $\sim 1 \mathrm{AU}$. When combined with the lower limit on its mass, the lower limit on the mass density is $6.5 \times 10^{21} M_{\odot} p c^{-3}$, which provides strong evidence favoring Sgr A* as a super-massive black hole.

Hyman et al. (2005) report a transient radio source, GCRT J1745-3009, which was detected during a moderately wide-field monitoring program of the Galactic Centre region at $0.33 \mathrm{GHz}$. The characteristics of its bursts are unlike those known for any other class of radio transient.

\subsection{Extragalactic Sources}

\subsubsection{Galaxies}

Minchin et al. (2005) report the detection in HI of what appears to be a dark halo that does not contain the expected bright galaxy. A galaxy with the observed velocity width would be expected to be 12 mag or brighter; however, deep CCD imaging has failed to turn up a counterpart down to a surface brightness level of $27.5 \mathrm{~B}$ mag $\operatorname{arcsec}^{-2}$. However, Bekki et al. (2005) argue that this object is not really a dark halo but most likely tidal debris from the nearby galaxy NGC 4254 .

Solomon \& vanden Bout (2005) have reviewed the sample of 36 detected galaxies that have molecular masses in the range of $4 \times 10^{9}$ to $1 \times 10^{11} M_{\odot}$ and star formation rates derived from their FIR luminosities in the range of 300 to $5000 M_{\odot} y^{-1}$. These objects are generally starbursts in centrally concentrated disks, sometimes, but not always, associated with active galactic nuclei.

Brunthaler et al. (2005) measured the angular rotation and proper motion of the Triangulum Galaxy (M33) with VLBI observations of two $\mathrm{H}_{2} \mathrm{O}$ masers on opposite sides of the galaxy. By comparing the angular rotation rate with the inclination and rotation speed, they obtain a distance of $730 \pm 168$ kiloparsecs. This distance is consistent with the most recent Cepheid distance measurement.

\subsubsection{Gamma Ray Bursts}

Radio astronomy played a key role in the elucidation of the nature of cosmic gamma ray bursts (Mészáros 2002; Weiler et al. 2002).

\subsubsection{Active Galaxies and Quasars}

A review on mega-masers in external galaxies was completed by Lo (2005). Drake et al. (2003) and Bassett et al. (2004) have questioned the so-called radio loud/quiet dichotomy by finding many "intermediate" radio galaxies in their samples. Merritt \& Ekers (2002) show evidence that binary super-massive black holes may be produced by 
galactic mergers as the black holes from the two galaxies fall to the center of the merged system and form a bound pair. They propose that the winged or X-type radio sources are galaxy pairs in which this merging has occurred.

\subsection{Cosmology}

A major development was the accurate measurement and interpretation of the anisotropies in the cosmic microwave background (Hu \& Dodelson 2002). The spectrum of amplitudes of temperature (or brightness) fluctuations expanded in multi-pole moments reported by the Wilkinson Microwave Anisotropy Probe (WMAP) satellite is a remarkable achievement that allowed accurate determination of the main cosmological constants (Bennett et al. 2003; Spergel et al. 2003). The detection of polarization in the cosmic microwave background (Kovac et al. 2002) confirmed the predictions of the standard theory.

There also significant advances in the measurement of the Sunyaev-Zeldovich effect in clusters of galaxies (Carlstrom et al. 2002; Lancaster et al. 2005). The realization that the first generation of stars (Bromm \& Larson 2004) may be detectable by means of radio observations of red-shifted atomic hydrogen triggered the development of ad hoc radio telescopes for this purpose and results may be obtained in a few years.

\section{Triennial Report of the Working Group on Historic Radio Astronomy (Wayne Orchiston)}

\subsection{Introduction}

This WG was formed at the 2003 General Assembly of the IAU as a joint initiative of Commissions 40 (Radio Astronomy) and 41 (History of Astronomy), in order to:

- assemble a master list of surviving historically-significant radio telescopes and associated instrumentation found worldwide;

- document the technical specifications and scientific achievements of these instruments;

- maintain an on-going bibliography of publications on the history of radio astronomy; and

- monitor other developments relating to the history of radio astronomy (including the deaths of pioneering radio astronomers).

The membership list of the WG contains the names of about one hundred astronomers who are active in the history of radio astronomy field or sympathetic to it.

\subsection{Progress Reports of the Working Group}

Annual Progress Reports were prepared in 2004 and 2005 and were published in the newsletter of Commission 41 and in the June 2004 and 2005 issues of the Journal of Astronomical History and Heritage. Copies also were submitted to Commission 40 and to the Presidents of Divisions X and XII.

\subsection{National Masterlists of Surviving Historically Significant Radio Telescopes}

WG members actively worked on national masterlists for Australia, Germany, the Netherlands, the United Kingdom and the USA, and a number of research papers were prepared documenting individual instruments or instruments and research associated with specific radio astronomy field stations.

\subsection{The Preservation and Destruction of Historically-Significant Radio Telescopes}

WG members lobbied Stanford University to rescind its decision to demolish the array of five 60-ft antennas at the field station off Highway 280 (California), and reported-with 
some dismay-the demolition of the twelve surviving Chris Cross dishes from the solar grating array at Fleurs, near Sydney.

\subsection{Research Projects by Working Group Members}

WG members who actively researched aspects of radio astronomical history during the triennium included Bruce Balick (USA), Ron Bracewell (USA), Jessica Chapman (Australia), Marshall Cohen (USA), Rod Davies (United Kingdom), Bob Duncan (Australia), Dave Green (United Kingdom), Miller Goss (USA), Alastair Gunn (United Kingdom), Richard Jarrell (Canada), Dave Jauncey (Australia), Ken Kellermann (USA), Bruce McAdam (Australia), Dick McGee (Australia), Doug Milne (Australia), Masaki Morimoto (Japan), Wayne Orchiston (Australia), Brian Robinson (Australia), Bruce Slee (Australia), Slava Slysh (Russia), Richard Strom (The Netherlands), Woody Sullivan (USA), Govind Swarup (India), Hugo Van Woerden (The Netherlands), John Whiteoak (Australia), and Richard Wielebinski (Germany).

\subsection{Bibliography of Publications on the History of Radio Astronomy}

An on-going list of publications in the history of astronomy field was maintained (and included in the WG Annual Reports). Arrangements were made to publish a succession of history of astronomy papers in 2005 and 2006 issues of the Journal of Astronomical History and Heritage.

\subsection{Conferences}

Since the 2003 General Assembly sessions on historic radio astronomy, the following conferences have featured sessions on the history of radio astronomy: (1) "The New Astronomy: Opening the Electromagnetic Window and Expanding our View of Planet Earth. A Meeting to Honor Woody Sullivan on His 60th Birthday" (Seattle, June 2004); (2) "Radio Astronomy at 70: From Karl Jansky to Microjansky" (Budapest, August 2004); (3) Fifth International Conference on Oriental Astronomy (Chiang Mai, Thailand, October 2004); (4) AAS Historical Astronomy Division Meeting (Cambridge, UK, September 2005). Members of the WG were instrumental in organizing all but the second of these.

\subsection{Planning for the Prague General Assembly}

The WG Committee has applied to hold four quarter-day meetings, so that members can discuss their latest research, with emphasis on the development of radio astronomy in Europe, and the status of radio astronomy worldwide fifty years ago when 'big science' first began to impact on radio astronomy.

\subsection{The End of an Era}

With sadness WG members noted the passing of the following pioneering radio astronomers: Semion Braude, Robert Hanbury Brown, Bob Duncan, Frank Gardner, Victor Hughes, Vladimir Kotelnikov, John D. Kraus, Harry Minnett, Christiaan Alexander (Lex) Muller, Grote Reber, Brian Robinson, Gordon Stanley, Hendrik Christoffel (Henk) van de Hulst, Kevin Westfold and Don Yabsley. Where these existed, relevant obituaries were included in the WG Annual Reports, and biographical details were provided for several of these individuals.

\subsection{Further Information}

For further details of Working Group activities during 2003-2005 see Orchiston, W. et al. (2004; 2005). 


\subsection{Members of the Working Group}

At present, this Working Group is constituted by:

Wayne Orchiston, Chair (Australia), Rod Davies (United Kingdom), Ken Kellermann (USA), Alain Lecacheux (France), Masaki Morimoto (Japan), Slava Slysh (Russia), Govind Swarup (India), Hugo Van Woerden (The Netherlands), Jasper Wall (Canada), and Richard Wielebinski (Germany)

\section{References}

Bassett, L. C., et al. 2004, AJ, 128, 523

Bekki, B. et al. 2005, MNRAS, 363, 21

Bennett, C. L., et al. 2003, ApJS, 148, 1

Bower, G. C., et al. 2002, ApJ, 571, 843

Bower, G. C., et al 2004, Science, 304, 704

Bromm, V. \& Larson, R. B. 2004, ARA\&A, 42, 79

Carlstrom, J. E., Holder, G. P., \& Reese, E. D. 2002, ARA\&A, 40, 643

Cameron, P. B. et al. 2005, Nature, 434, 1112

Chatterjee, S. et al. 2005, ApJ, 630, L61

Churchwell, E. 2002, ARA\&A, 40, 27

Dougherty, S. M. et al. 2005, ApJ, 623, 447

Drake, C. L., et al. 2003, AJ, 126, 2237

Evans, N. J. et al. 2005, ApJ, 626, 919

Fender, R. \& Belloni, T. 2004, ARA\&A, 42, 317

Hankins, T. H. et al. 2003, Nature, 422, 141

Harvey, D. W. A., et al. 2002, AJ, 123, 3325

$\mathrm{Hu}, \mathrm{W}$. \& Dodelson, S. 2002, ARA\&A, 40, 171

Hyman, S. D. et al. 2005, Nature, 434, 50

Kovac et al. 2002, Nature, 420, 772

Kuan, Y.-J., et al. 2003, ApJ, 593, 848

Lancaster, K. et al. 2005, MNRAS, 359, 16

Lo, K. Y. 2005, ARA\&A, 43, 625

Loinard, L. et al. 2005, ApJ, 619, L179

Lyne, A. G. et al. 2004, Science, 303, 1153

Marscher, A. P. et al. 2002, Nature, 417, 625

Merritt, D. \& Ekers, R. D. 2002, Science, 297, 1310

Mészáros, P. 2002, ARA\&A, 40, 137

Minchin, R. et al. 2005, ApJ, 622, L21

Orchiston, W. et al., 2004. The IAU Historic Radio Astronomy Working Group. 1. Progress Report. Journal of Astronomical History and Heritage, 7, 53-56

Orchiston, W. et al., 2005. The IAU Historic Radio Astronomy Working Group. 2. Progress Report. Journal of Astronomical History and Heritage, 8, 65-69.

Patel, N. et al. 2005, Nature, 437, 109

Ransom, S. M. et al. 2005, Science, 307, 892

Rodríguez, L. F. 2005, in Massive star birth: A crossroads of Astrophysics, IAU Symposium Proceedings of the international Astronomical Union 227, Held 16-20 May, Italy, edited by Cesaroni, R.; Felli, M.; Churchwell, E.; Walmsley, M. Cambridge: Cambridge University Press, 2005., pp. 120-127

Rodríguez, L. F. et al. 2005, ApJ, 627, L65

Shen, Z.-Q. et al. 2005, Nature, 438, 62

Shirley, Y. L., et al. 2002, ApJ, 575, 337

Snyder, L. E. et al. 2005, ApJ, 619, 914

Solomon, P. M. \& vanden Bout, P. A. 2005, ARA\&A, 43, 677

Spergel, D. N. et al. 2003, ApJS, 148, 175

Trimble, V. \& Aschwanden, M. J. 2004, PASP, 116, 187

Weiler, K. W., Panagia, N., Montes, M. J., \& Sramek, R. A. 2002, ARA\&A, 40, 387 